healthcare outcomes. At present there is less emphasis on developing leadership skills compared to other aspects of training

Aim To understand anaesthetic trainees' experience of leadership and management and how best to develop these skills during training. Ultimately, this led to the implementation of a leadership and development passport (adapted from the Faculty of Medical Leadership and Management leadership passport) amongst anaesthetic trainees.

Method An electronic survey was sent to all anaesthetic trainees in our trust. It consisted of ten questions regarding leadership opportunities, at what level of training to introduce leadership and management skills and how best to achieve these goals.

Results In total 22 trainees responded. 95\% thought leadership and management skills were important with 59\% thinking they should be incorporated throughout training. 95\% of trainees have had ideas in improving patient care or the working environment, however, $77 \%$ have not had these sustainably implemented. Multiple barriers were identified with lack of opportunity and lack of time being the top two.

A vast majority, 82\%, thought that the leadership passport would be a useful tool.

Conclusion Trainees are meeting barriers having their improvement projects implemented. Despite increasing emphasis on doctors' leadership and management skills by the GMC, the practicalities of leadership development during training remains unclear. This leadership passport aims to empower trainees to sustain their ideas and to support them in developing leadership and management skills. We are confident that this will be an invaluable tool in developing the leadership skills of tomorrow's doctors.

\section{Quality improvement project}

\section{IMPROVING BASELINE MEASUREMENT OF BLOOD GLUCOSE AND CHOLESTEROL LEVELS IN ACUTE STROKE PATIENTS: A QUALITY IMPROVEMENT PROJECT}

Ayesha Khan*, Shruti Dorai*. Queen Elizabeth the Queen Mother Hospital, UK

\subsection{6/leader-2020-FMLM.159}

Introduction Hypercholesterolaemia and diabetes are established modifiable risk factors for cerebrovascular disease. A baseline audit carried out on an acute stroke ward in Kent showed variability in blood tests being requested on admission for suspected stroke patients, in particular glucose and lipid profile.

Aim To ensure that atleast $80 \%$ of patients admitted to the stroke ward with suspected stroke have blood glucose and cholesterol levels measured on admission over an 18-week period.

Methods The percentage of patients with suspected stroke on the ward who had blood glucose and/or total cholesterol levels requested on admission was measured weekly. 3 interventions were introduced in the form of Plan Do Study Act cycles: educational email to doctors regarding the assessment of stroke patients and mandatory admission blood tests, adaptation of the 'Stroke Admission Clerking Proforma' to include a reminder of bloods to be requested and production of an all-inclusive 'Stroke bloods panel' on the online system for requesting bloods.
Results At baseline, an average of 30\% and 34\% of patients had glucose and cholesterol levels requested on admission, respectively, which increased to $43 \%$ and $40 \%$ respectively, after the email. This increased to $71 \%$ and $61 \%$ after the introduction of the proforma, and after the final intervention, on average, $82 \%$ and $85 \%$ of patients had glucose and cholesterol levels requested on admission, respectively. The results showed non-random variation.

Conclusions We achieved our aim of ensuring that more than $80 \%$ of patients with acute stroke had both glucose and cholesterol levels requested on admission. The stroke proforma and the bloods panel were the most effective interventions. The changes were implemented in another hospital within the same Trust. We anticipate greater compliance with NICE and Trust guidelines regarding appropriate and timely prescription of antidiabetic and cholesterol-lowering medications for secondary prevention.

\section{Leading innovation and improvement}

\section{TURNING A CRISIS INTO AN OPPORTUNITY FOR GENERAL PRACTICE TEAMS IN NI}

Louise Sands, Maura Corry, Siobhan McEntee, Ursula Mason, Patrick Stirling. GPNI Team, UK

\subsection{6/leader-2020-FMLM.160}

The emergence of COVID-19 saw a seismic change in General Practice with significant clinical, operational and educational challenges across the whole of the primary care team. Information and guidance from multiple different sources emerged at an overwhelming pace for practitioners. There was no robust way of cascading critical information to individuals. Shielded, remote and sessional health care professionals in particular lacked access to sensitive Health and Social Care Board (HSCB) information.

It was evident that there was a need for a centralised information platform for professionals. This would provide easily accessible, accurate and up to date information on service changes, operational and clinical guidance as well as legislative changes

An MDT working group, with representatives from all professions including trainees was formed to design a central knowledge repository for the whole primary care team. This ensured all practitioners had access to the latest information, equipping them to deliver high quality care during the pandemic.

From concept to website launch took just three weeks, with a live web-based educational programme starting just one week later and acting as a catalyst for enhanced primary and secondary care understanding and communication.

There are over 150 attendees at weekly live ZOOM educational events, with the programme reflecting learning needs across the whole primary care team

Website analytics confirm 'Pageviews' >100,000 and 'users' $>7,500$ and rising with a global audience.

A crisis can bring exciting opportunities and a highly effective team can be created from conception in less than 4 weeks with a shared vision, enthusiasm and determination to make it work. 
Collaborative learning between GPs, fledgling MDTs, and across the primary-secondary care interface has united colleagues around a shared purpose, starting solution-focused conversations.

\section{OUTCOMES OF THE ELECTRONIC MEDICAL TAKE LIST - IMPROVING STAFF EXPERIENCE, PATIENT SAFETY AND ROSTERING}

Alvin Shrestha, Alexia Pisinou, Craig Burke, Troy Haddon. Croydon University Hospital, UK; 3, 4 Patienteer

\subsection{6/leader-2020-FMLM.161}

Aims and Methods The 'medical take' is a demanding process, whereby doctors review and manage acute admissions. We created a task force group and collaborated with Patienteer, a patient tracking software that can pull real-time patient information from our electronic medical record system Cerner, in order to improve this experience for our staff and ultimately improve patient care.

A bespoke electronic medical take list was which allowed us to overcome previously identified issues:

- Patient details were automatically added to the list (removing the potential for transcription errors)

- Multiple user access

- Live location

- Live NEWS2 scores

Thereafter, 6 months' worth of data was analysed from February to September 2019.

Results Doctors were surveyed through an anonymised online questionnaire, with 27 respondents who had used both the old Excel list and the new Patienteer list. 89\% found Patienteer to be improve efficiency. Two-thirds overall found it helped prioritise clinical need. $78 \%$ found patient details were captured more accurately with Patienteer. Accessibility was also thought to have improved, with $85 \%$ favouring Patienteer as being 'easier' or 'much easier' to access. 59\% thought Patienteer had decreased their workload. 96\% would overall, recommend the new Patienteer medical take list.

Through 6344 patient encounters, various outcomes were reviewed. There was a significant inverse correlation between NEWS2 scores and time taken for a medical doctor review: as NEWS2 score increased, patients were seen earlier. The time taken to be seen in minutes was expressed as = 4.24621*NEWS +133.031 , where NEWS is the NEWS2 score $(\mathrm{p}=0.0002397)$.

Conclusion The survey suggested the Patienteer medical take list had made the acute medical take experience more efficient and decreased the workload. There is also a suggestion that patient care is improved through the display of the NEWS2 score, allowing doctors to prioritise sicker patients earlier.

\section{OPTIMISING THE CLINICAL PATHWAY FOR PLACENTAL HISTOLOGY}

${ }^{1}$ Nazia Din, ${ }^{1}$ Nicole Gentles, ${ }^{1}$ Benjamin Allison, ${ }^{1}$ Maria Hickland, ${ }^{2}$ Jilly Lloyd, ${ }^{2}$ Adam D Jakes. 'King's College London, UK; ${ }^{2}$ Guy's and St. Thomas' NHS Foundation Trust, London, UK

10.1136/leader-2020-FMLM.162
The Royal College of Pathologists (RCPATH) sets criteria for requesting placental histological examination which allows identification of pathological processes contributing to/causing an adverse obstetric outcome. Maternal intrapartum pyrexia is an essential criterion but is not part of the Guy's and St. Thomas' Trust (GSTT) guideline. There is no current baseline data on this at GSTT.

Aim To review current practice of requesting placental histology and amend trust guidelines to follow national recommendations.

Birth records from June 2019 were reviewed showing $8 \%(47 / 569)$ of deliveries were eligible for placental histology as per trust criteria but only 60\%(28/47) were requested. A survey demonstrated $4 \%$ of staff were able to correctly identify all criteria for histology and there was confusion regarding formalin use. Neonatologists and pathologists were contacted to identify views on the usefulness of placental histology and the effect of adopting national criteria.

The pathologists confirmed all placentas should be sent in formalin unless specified by the obstetric team. The intrapartum infection guideline was amended to include requesting placental histology after approval by the intrapartum committee. The obstetric theatre posters were amended to include the extended criteria. A training presentation was emailed to all staff on the procedure for requesting placental histology.

The trust guidelines were successfully amended to include RCPATH criteria for placenta histology. Birth records from January 2020 identified 13\%(63/487) of deliveries were eligible for placental histology, but only 56\%(35/63) were requested.

The trust guideline for requesting placental histology now follows national recommendations. This may provide answers for adverse obstetric outcomes and reduce trust medicolegal costs. Unfortunately, no improvement in placental histology requests was demonstrated. Exploring potential barriers to improvement will be part of the next change cycle.

\section{QUALITY IMPROVEMENT PROJECT ON COMMUNITY INDUCTION FOR FOUNDATION YEAR 2 DOCTORS}

${ }^{1} \mathrm{H}$ Minali Perera, ${ }^{2}$ Victoria Apel, ${ }^{2}$ Katie Chu. ${ }^{1}$ Princess Alexandra Hospital, Harlow; ${ }^{2}$ Epsom and St Helier Hospital NHS Trust, Surrey

\subsection{6/leader-2020-FMLM. 163}

Foundation doctors regularly rotate through different specialties as part of their training. Inductions to these new placements can vary in delivery and effectiveness. High quality induction is a recommendation of the Academy of Medical Royal Colleges for safe trainee changeover.

Aim To improve the induction process for Foundation Year 2 doctors (FY2s) in community placements at Epsom and St Helier Trust.

A survey of 10 questions, devised from the British Medical Association guidance on adequate induction, was circulated to FY2 doctors $(n=10)$ in community placements (GP and Psychiatry) between 4th August - 4th December 2019. Induction booklets individualised to each placement were created by 